\title{
Compressão do nervo ulnar na região do cotovelo - síndrome do túnel cubital: revisão da literatura
}

\section{Compression of the Ulnar Nerve in the Elbow Region - Cubital Tunnel Syndrome: Review of the Literature}

\author{
Roger Neves Mathias ${ }^{1}$ Mário Gilberto Siqueira ${ }^{2}$ Roberto Sérgio Martins ${ }^{2}$ Hugo Sterman Neto ${ }^{2}$ \\ Luciano Foroni $^{2}$ Manoel Jacobsen Teixeira ${ }^{3}$ \\ ${ }^{1}$ Residente, Disciplina de Neurocirurgia Faculdade de Ciências \\ Médicas da Universidade Estadual de Campinas (FCM-Unicamp), \\ Campinas, Brasil \\ 2 Divisão de Cirurgia dos Nervos Periféricos, Disciplina de \\ Neurocirurgia, Hospital das Clínicas da Faculdade de Medicina da \\ Universidade de São Paulo (HC-FM-USP), São Paulo, Brasil \\ 3 Professor Titular da Divisão de Neurocirurgia do HC-FMUSP, São \\ Paulo, Brasil \\ Arq Bras Neurocir 2015;34:128-133. \\ Address for correspondence Roger Neves Mathias, MD, \\ (e-mail: mathias94@hotmail.com).
}

\section{Resumo}

Palavras-Chave

- síndrome do túnel cubital

- neuropatia compressiva

- nervo ulnar
A síndrome do túnel cubital é responsável pela neuropatia do nervo ulnar, sendo superada em frequência apenas pela síndrome do túnel do carpo. O nervo ulnar apresenta anatomia complexa podendo sofrer compressão em distintos pontos ao longo de seu trajeto, por isso o entendimento das nuances clínicas e da anatomia pormenorizada assim como da técnica cirúrgica meticulosa torna-se essencial no tratamento desta patologia.

The cubital tunnel syndrome is responsible for the ulnar nerve neuropathy, this condition is surpassed in frequency only by carpal tunnel syndrome. The ulnar nerve has complex anatomy and may suffer compression at different points along its path, so understanding the clinical nuances and detailed anatomy as well as meticulous surgical technique becomes essential in the treatment of this pathology.

\section{Introdução}

A neuropatia do nervo ulnar é a segunda mais frequente nos membro superiores, sendo superada apenas pela síndrome do túnel do carpo. ${ }^{1,2}$ Apesar de poder sofrer compressão em pontos distintos em seu trajeto, o cotovelo é o local mais comum. ${ }^{3}$ Este artigo tem como objetivo discutir a anatomia do nervo ulnar no cotovelo assim como sinais e sintomas decorrentes da síndrome do túnel cubital, além de esclarecer as modalidades terapêuticas e os avanços técnicos atuais tanto nos casos primários quanto nas recidivas. received

August 25, 2014

accepted

March 31, 2014
DOI http://dx.doi.org/ $10.1055 / \mathrm{s}-0035-1554049$ ISSN $0103-5355$.
Copyright $\odot 2015$ by Thieme Publicações License terms Ltda, Rio de Janeiro, Brazil
(ब) $\Theta \circledast$ 


\section{Anatomia}

Ao deixar o compartimento flexor do braço e entrar no compartimento extensor, o nervo ulnar perfura o septo intermuscular medial $8 \mathrm{~cm}$ proximal ao epicôndilo medial onde pode sofrer compressão. Aqui o nervo pode ser "coberto" por um espessamento da fáscia braquial e por fibras da cabeça medial do tríceps em direção ao septo muscular medial formando uma espécie túnel (arcada de Stuthers, - Fig. 1), configurando outro ponto de possível compressão, quando existente. ${ }^{4}$ Neste ponto o nervo ulnar segue um trajeto descendente entre a cabeça medial do músculo tríceps e o músculo braquial, acompanhado pela artéria ulnar colateral superior até o nível do cotovelo, sem dar ramos importantes. ${ }^{5}$ O nervo então tem um curso posterior ao epicôndilo medial e medial ao olécrano quando entra no túnel cubital. 0 teto deste é formado pelo ligamento arqueado de Osborne; trata-se de uma banda espessa entre as cabeças umeral e ulnar do músculo flexor ulnar do carpo (MFUC). O assoalho do túnel cubital é formado pelo ligamento colateral do cotovelo, pela cápsula articular do cotovelo e pelo olécrano. Ao sair do túnel o nervo ulnar tem um trajeto profundo às cabeças ulnar e umeral do MFUC. Na região do cotovelo o nervo ulnar pode sofrer compressão em cinco pontos principais: (1) na arcada de Struthers, (2) no septo intermuscular medial, (3) no epicôndilo medial, (4) no túnel cubital pelo ligamento de Osborne e (5) pela fáscia do grupo muscular flexora-pronadora do antebraço. ${ }^{3,6}$ Entre estes, o local mais frequente de compressão é o túnel cubital, ${ }^{7}$ por isso exploração proximal e distal dessa estrutura é mandatória para efetuar-se descompressão efetiva.

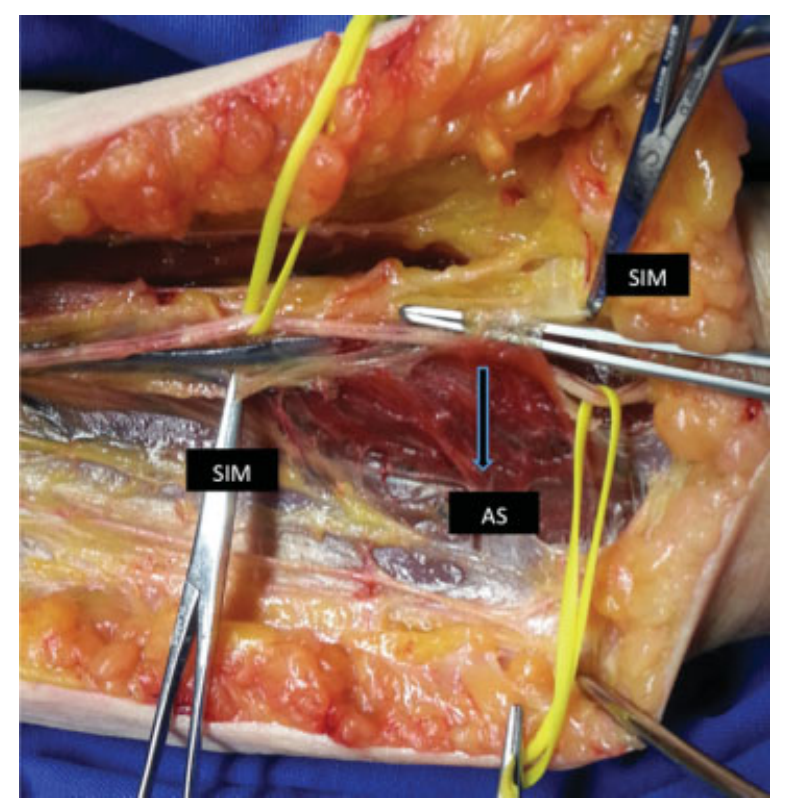

Fig. 1 Dissecção do nervo ulnar em cadáver fresco. Pinças reparando o septo intermuscular (SIM). Nervo ulnar perfurando o SIM passando do compartimento plexor para o compartimento extensor do braço. Fibras da cabeça medial do músculo tríceps conectando-se ao SIM, arcada de Struthers (AS).
Outro ponto importante é a relação que o ramo posterior do nervo cutâneo medial do antebraço tem com o epicôndilo medial, que torna este ramo particularmente propenso à lesão iatrogênica durante a cirurgia de descompressão do nervo ulnar. Essa lesão é responsável por uma das causas de dor pós-operatória. ${ }^{8}$ Lowe et al. ${ }^{9}$ demostraram que em $61 \%$ dos casos o ramo posterior do nervo cutâneo medial do antebraço cruza a linha de incisão.

\section{Apresentação Clínica}

\section{História e Antecedentes}

Pacientes que necessitam fazer flexão do antebraço frequentemente são mais susceptíveis à compressão do nervo ulnar no cotovelo (telefonistas e trabalhadores com máquinas vibratórias). Outro fator predisponente é a prática de esportes que demandam arremeço com flexão forçada acima da cabeça e rápida aceleração (beisebol, golfe). ${ }^{10}$

Doenças sistêmicas devem ser lembradas diante da suspeita de neuropatia do nervo ulnar, entre as principais estão diabetes melito, hipotireoidismo e hanseníase.

\section{Sinais e Sintomas}

O examinador deve questionar o paciente quanto ao inicío de parestesia e hipoestesia no território do nervo ulnar (quarto e quinto dedos), geralmente com piora noturna. Em casos mais avançados, fraqueza e atrofia da musculatura intrínseca da mão é evidente (-Fig. 2). A garra ulnar (sinal de Duchenne) por fraqueza do terceiro e quarto lumbricais e fraqueza do terceiro interósseo palmar responsável pela adução do quinto dedo leva ao sinal de Wartenburg (paciente com quinto dedo ligeiramente abduzido quando em repouso por déficit na adução). Outro sinal importante é o sinal de Froment. Pede-se para o paciente segurar um pedaço de papel entre o polegar e o indicador. Quando a função motora do nervo ulnar está íntegra, o paciente é capaz de manter a preensão usando o músculo adutor do polegar. Se o nervo ulnar está acometido, a preensão passa a ser compensada pelo músculo flexor longo do polegar que tem inervação do nervo mediano (-Fig. $\mathbf{3}$ ).

É importante questionar se os sintomas são intermitentes ou constantes, já que quando há sintomas transitórios isso pode denotar compressão focal e isquemia transitória e pode haver restauração completa da função do nervo com intervenção, diferentemente de sintomas constantes, quando a lesão já está estabelecida, e o prognóstico da intervenção é incerta. $^{3}$

Testes provocativos podem auxiliar no diagnóstico. 0 teste de Tinel ao longo da trajetória do nervo tem uma sensibilidade de $70 \%$ enquanto a flexão isolada do cotovelo apresenta sensibilidade em torno de $75 \%$. Já a compressão por 60 segundos sobre o túnel cubital apresenta sensibilidade de $89 \%$, porém a associação de métodos flexão do cotovelo e compressão passa a ter $98 \%$ de sensibilidade diagnóstica. ${ }^{11}$

Inspeção dinâmica para possível subluxação do nervo ulnar sobre o epicôndilo medial é importante, uma vez que pode mudar a conduta cirúrgica. 


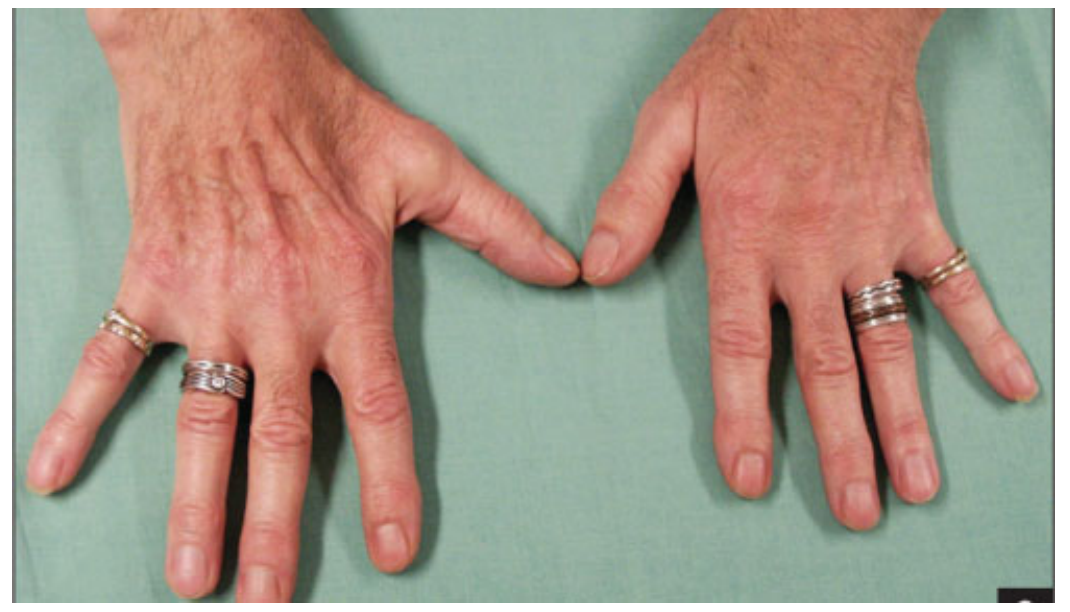

Fig. 2 Caso avançado de síndrome do túnel cubital à direita. Atrofia da musculatura intrínseca da mão inervada pelo ulnar. Atrofia hipotenar e dos interósseos dorsais. Fonte: Damert et al. ${ }^{25}$
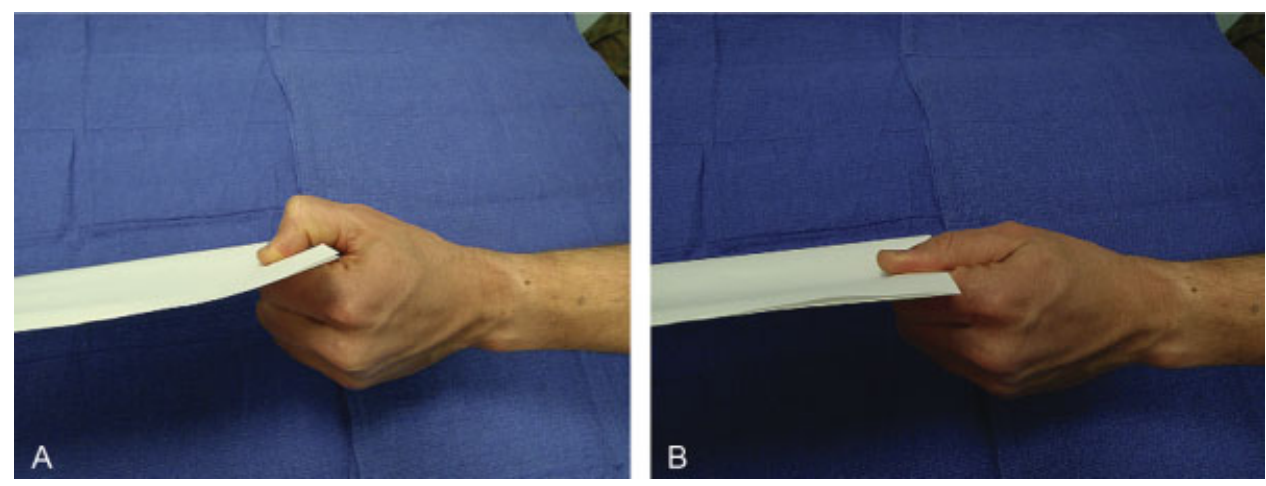

Fig. 3 Sinal de Fromet: quando solicitado a segurar um papel entre o polegar e o indicador, o paciente com nervo ulnar íntegro é capaz de usar o músculo adutor do polgar (A); quando há déficit no território do ulnar, o paciente faz compensação com o músculo flexor longo do hálux, território de inervação do nervo mediano (B). Fonte: Kroonen. ${ }^{12}$

\section{Classificação Clínica de McGowan}

Grau I: neuropatia sensitiva isolada

Grau II: neuropatia sensitiva e motora sem atrofia muscular

Grau III: neuropatia sensitiva e motora com atrofia muscular

\section{Exames Complementares}

Radiografia de cotovelo: importante para excluir deformidade, osteófitos que causem compressão e instabilidade de corrente de trauma prévio.

Ultrassonografia: exame dinâmico, pode evidenciar compressão e subluxação do nervo ulnar com a flexão do antebraço. Análise da área em cortes seccionais ultrassonográficos é estatisticamente menor em paciente com compressão. ${ }^{13}$

Eletroneuromiografia (ENMG): importante para confirmar o diagnóstico clinico e localizar o ponto de compressão. Queda na velocidade de condução do nervo ulnar na região do cotovelo abaixo de $50 \mathrm{~m} / \mathrm{s}$ é diagnóstico. Pacientes com sintomas leves a moderados a ENMG podem ser falso negativo, porém em paciente com sintomas graves, a ENMG pode ser útil como ferramenta prognóstica da recuperação muscular e do nervo após o tratamento. ${ }^{12}$

\section{Tratamento Conservador}

Casos leves a moderados podem ser tratados de forma conservadora devido ao potencial de regeneração espontânea desde que os sintomas sejam leves ou intermitentes e os fatores provocativos possam ser eliminados. Paciente com sintomas constantes e atrofia muscular geralmente necessitam intervenção cirúrgica.

O tratamento conservador consiste em readequação de atividades cotidianas que exijam hiperflexão do antebraço, fisioterapia e uso de tala ou anteparo que limite a flexão do cotovelo em até 45 graus (principalmente durante o sono). Svernlov et al notaram que $89,5 \%$ dos pacientes com lesão leve a moderada tratados de forma conservadora obtiveram melhora sintomática, não havendo diferença estatística entre grupos que usaram tala daqueles que apenas foram orientados sobre a doença e seus desencadeantes. ${ }^{14}$ 


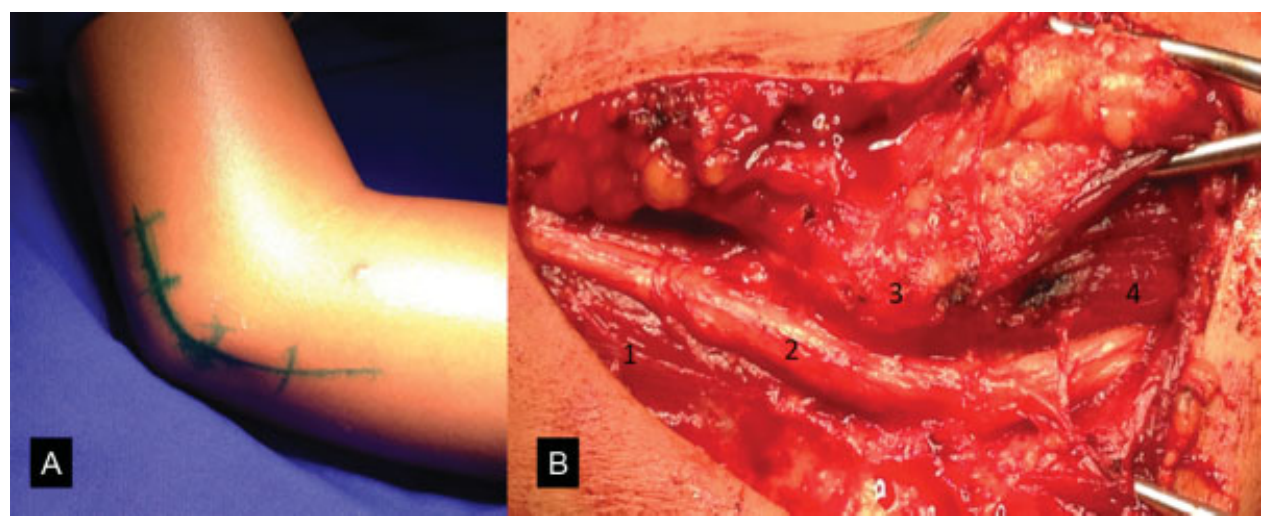

Fig. 4 (A) Incisão cutânea para descompressão do nervo ulnar na região do cotovelo. (B) Descompressão in situ. (1) Cabeça medial do músculo tríceps; (2) nervo ulnar no sulco retroepicondilar; (3) epicôndilo medial; (4) músculo flexor ulnar do carpo (cabeça umeral).

\section{Tratamento Cirúrgico}

Está indicado a paciente com déficit motor no território do nervo ulnar ou quando houve falha no tratamento conservador. ${ }^{14}$ Há várias técnicas para descompressão do nervo ulnar na região do cotovelo e não há controvérsias na literatura a respeito da melhor técnica. ${ }^{15}$ As técnicas mais comuns são a descompressão in situ, transposição subcutânea, transposição intramuscular, transposição submuscular e epicondilectomia medial. Mais recentemente, avanços endoscópicos permitiram a descompressão do nervo mediano com o uso dessa modalidade terapêutica.

\section{Descompressão in situ}

Uma incisão cutânea arciforme de $6-10 \mathrm{~cm}$ ao longo do trajeto do nervo ulnar na região do cotovelo é feita. Durante a dissecção superficial deve-se ter precaução para evitar lesão do ramo cutâneo medial do antebraço. É importante promover dissecção tanto proximal no ligamento de Osborne quanto distal nas fáscias superficiais e profundas do MFUC. Evita-se dissecção circunferencial do nervo, mantendo seu tecido conectivo a fim de evitar subluxação do nervo (-Fig. 4).

Evidências mostram que essa técnica é eficaz no tratamento da compressão e com baixa taxa de recorrência, quando comparada à transposição anterior, ${ }^{16,17}$ deixando esta última para casos de sintomas recorrentes com melhores respostas. ${ }^{18,19}$

\section{Transposição Anterior}

Flexão do antebraço gera estiramento do nervo ulnar e diminui o volume do túnel cubital, piorando a compressão e consequentemente causando isquemia do nervo. 0 objetivo da transposição anterior é levar o nervo anteriormente à linha de flexão do cotovelo, diminuindo assim a tensão sobre ele. No entanto a dissecção do nervo pode acarretar comprometimento em seu suprimento vascular. ${ }^{20}$ Deve-se tomar cuidado para não criar novos pontos de compressão tanto distal quando proximal após a transposição, para isso uma incisão cutânea maior é feita, e inicialmente realiza-se descompressão in situ. Em seguida o septo intermuscular e a arcada de Struthers ${ }^{4}$ (quando presente) devem ser removidos, evitando-se pontos de compressão proximais. O nervo é então dissecado circunferencialmente com auxílio de uma tração suave de um vessel loop, sendo então transposto anteriormente ao epicôndilo medial. Deve-se tomar cuidado para preservar os ramos que vão para as cabeças musculares de flexor ulnar do carpo. O nervo pode ser sobreposto tanto por um coxim de tecido subcutâneo quanto criado um novo leito profundo à massa flexora-pronadora (-Fig. 5), devendo nesse caso atentar-se para lesão de nervo mediano durante a dissecção. Nenhuma dessas técnicas mostrou-se estatisticamente superior à descompressão in situ. ${ }^{17,21}$

\section{Epicondilectomia Medial}

Outra técnica para tratamento de neuropatia do nervo ulnar associada a descompressão in situ. A resseção completa do epicôndilo medial pode levar à instabilidade da articulação do cotovelo. Dessa forma, mantém-se a massa flexora-pronadora aderida ao epicôndilo. Essa técnica não se demonstrou superior à transposição subcutânea anterior. ${ }^{22}$

\section{Descompressão Endoscópica}

Técnica desenvolvida em 1995 em que uma pequena incisão sobre o sulco retroepicondilar é realizada e com auxílio de dissecção romba, afastadores subcutâneos e tesoura endoscópica, procede-se a lise neural de pontos de constrição. Esse tipo de técnica é efetiva no alívio dos sintomas e melhora neurológica evidenciados por ENMG. ${ }^{23}$ A comparação entre técnica endoscópica e descompressão in situ mostrou menor dor pós-operatória e maior satisfação de forma estatisticamente significante no grupo tratado por endoscopia, porém não houve diferença nos resultados objetivos da cirurgia. ${ }^{24}$

\section{Falha Terapêutica}

Fatores biológicos, diagnósticos e técnicos estão associados à falha terapêutica. Os dois últimos podem ser minimizados 


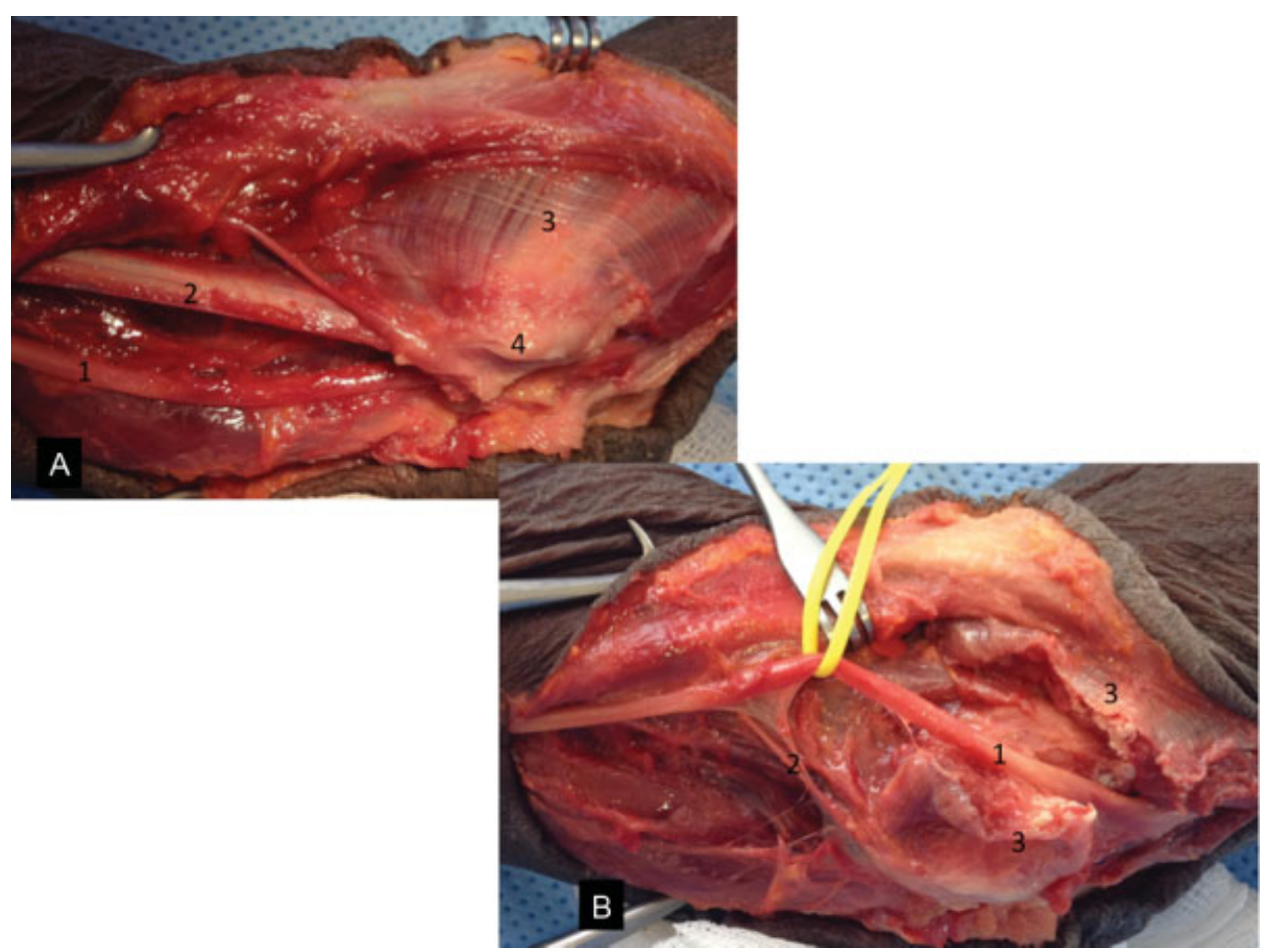

Fig. 5 (A) Ilustração da técnica cirúrgica de transposição anterior submuscular em cadáver congelado. (1) Nervo ulnar após lise do tecido conectivo; (2) septo intermuscular medial que necessita ser ressecado antes da transposição; (3) grupo muscular flexor-pronador; (4) epicôndilo medial. (B) Transposição anterior do nervo ulnar submuscular em cadáver. (1) Nervo ulnar transposto anteriormente ao epicôndilo medial; (2) ramo motor para a cabeça ulnar do músculo flexor ulnar do carpo; (3) grupo muscular flexor pronador seccionado.

pelo cirurgião, porém o primeiro não. Fibrose perineural, cicatriz e lesão neural pré-operatória devem ser detectadas, e o aconselhamento do paciente sobre as expectativas do tratamento é fundamental.

Recidiva dos sintomas e exames eletroneuromiográficos seriados mostrando nova queda na velocidade de condução do nervo ulnar são as principais bases para a indicação de reabordagem. No tocante a técnica operatória empregada, a transposição submuscular é a que mais tem suporte pela literatura (série de casos), porém não houve superioridade quando comparada com outras técnicas em um estudo prospectivo randomizado. Nellans et al advogam a descompressão do nervo ulnar de maneira efetiva e completa e a transposição anterior como uma opção à disposição do cirurgião. $^{19}$

\section{Referências}

1 Bozentka DJ. Cubital tunnel syndrome pathophysiology. Clin Orthop Relat Res 1998;(351):90-94

2 Coppieters MW, Bartholomeeusen KE, Stappaerts KH. Incorporating nerve-gliding techniques in the conservative treatment of cubital tunnel syndrome. J Manipulative Physiol Ther 2004;27(9): 560-568

3 Palmer BA, Hughes TB. Cubital tunnel syndrome. J Hand Surg Am 2010;35(1):153-163

4 Siqueira MG, Martins RS. The controversial arcade of Struthers. Surg Neurol 2005;64(Suppl 1):S1, 17-20, discussion S1, 20-21

5 Siqueira MG. Martins RS Anatomia Cirúrgica das Vias de Acesso aos Nervos Periféricos, Dilivros, 2006 ISBN 85-86703-25-7
6 Bruno W, Tsai T. Minimally invasive release of the cubital tunnel. Oper Tech Plast Reconstr Surg 2003;9(4):131-137

7 Ochiai N, Honmo J, Tsujino A, Nisiura Y. Electrodiagnosis in entrapment neuropathy by the arcade of Struthers. Clin Orthop Relat Res 2000; ((378):129-135

8 Dellon AL, MacKinnon SE. Injury to the medial antebrachial cutaneous nerve during cubital tunnel surgery. J Hand Surg [Br] 1985;10(1):33-36

9 Lowe JB III, Maggi SP, Mackinnon SE. The position of crossing branches of the medial antebrachial cutaneous nerve during cubital tunnel surgery in humans. Plast Reconstr Surg 2004; 114(3):692-696

10 Cutts S. Cubital tunnel syndrome. Postgrad Med J 2007;83(975): 28-31

11 Novak CB, Lee GW, Mackinnon SE, Lay L. Provocative testing for cubital tunnel syndrome. J Hand Surg Am 1994;19(5):817-820

12 Kroonen LT. Cubital tunnel syndrome. Orthop Clin North Am 2012;43(4):475-486

13 Wiesler ER, Chloros GD, Cartwright MS, Shin HW, Walker FO. Ultrasound in the diagnosis of ulnar neuropathy at the cubital tunnel. J Hand Surg Am 2006;31(7):1088-1093

14 Svernlöv B, Larsson M, Rehn K, Adolfsson L. Conservative treatment of the cubital tunnel syndrome. J Hand Surg Eur Vol 2009; 34(2):201-207

15 MacKinnon S, Novak C. Compression neuropathies-cubital tunnel syndrome. In: Green D, Hotchkiss R, Wolfe S, Pederson W, eds. Green's operative hand surgery. 5th ed. New York: Elsevier; 2005: 1023-1029

16 Gervasio O, Gambardella G, Zaccone C, Branca D. Simple decompression versus anterior submuscular transposition of the ulnar nerve in severe cubital tunnel syndrome: a prospective randomized study. Neurosurgery 2005;56(1):108-117, discussion 117

17 Nabhan A, Ahlhelm F, Kelm J, Reith W, Schwerdtfeger K, Steudel WI. Simple decompression or subcutaneous anterior 
transposition of the ulnar nerve for cubital tunnel syndrome. J Hand Surg [Br] 2005;30(5):521-524

18 Goldfarb CA, Sutter MM, Martens EJ, Manske PR. Incidence of reoperation and subjective outcome following in situ decompression of the ulnar nerve at the cubital tunnel. J Hand Surg Eur Vol 2009;34(3):379-383

19 Nellans K, Tang P. Evaluation and treatment of failed ulnar nerve release at the elbow. Orthop Clin North Am 2012;43(4): 487-494

20 Ogata K, Manske PR, Lesker PA. The effect of surgical dissection on regional blood flow to the ulnar nerve in the cubital tunnel. Clin Orthop Relat Res 1985;(193):195-198

21 Macadam SA, Gandhi R, Bezuhly M, Lefaivre KA. Simple decompression versus anterior subcutaneous and submuscular trans- position of the ulnar nerve for cubital tunnel syndrome: a metaanalysis. J Hand Surg Am 2008;33(8):1314.e1-1314.e12

22 Baek GH, Kwon BC, Chung MS. Comparative study between minimal medial epicondylectomy and anterior subcutaneous transposition of the ulnar nerve for cubital tunnel syndrome. J Shoulder Elbow Surg 2006;15(5):609-613

23 Hoffmann R, Siemionow M. The endoscopic management of cubital tunnel syndrome. J Hand Surg [Br] 2006;31(1):23-29

24 Watts AC, Bain GI. Patient-rated outcome of ulnar nerve decompression: a comparison of endoscopic and open in situ decompression. J Hand Surg Am 2009;34(8):1492-1498

25 Damert HG, Altmann S, Infanger M, Kraus A. Operative decisions for endoscopic treatment of cubital tunnel syndrome. Orthopedics 2013;36(5):354-359 